\title{
Retracted: Light Chain Glomerulopathy Causing Kidney Failure in Renal Cell Carcinoma
}

Article published 09/09/2021 Retracted 12/06/2021
Khashayar Farzam ${ }^{1}$, Keyan Zarei ${ }^{2}$

1. Family Medicine, University of Iowa Hospitals and Clinics, Iowa City, USA 2. Internal Medicine, University of Iowa Hospitals and Clinics, Iowa City, USA

Corresponding author: Khashayar Farzam, khashayar.f@hotmail.com

Corresponding author: Khashayar Farzam

1. Family Medicine, University of lowa Hospitals and Clinics, lowa City, USA 2. Internal Medicine, University of lowa Hospitals and Clinics, lowa City, USA

How to cite this retraction

Farzam K, Zarei K (December 06, 2021) Retraction: Light Chain Glomerulopathy Causing Kidney Failure in Renal Cell Carcinoma. Cureus 13(12): r38. doi:10.7759/cureus.r38

\section{Retraction Notice}

This article has been retracted at the request of the submitting author, Khashayar Farzam, as the University of Iowa Hospitals \& Clinics patient consent procedure was not properly followed by the authors when preparing this manuscript. Written consent was required from the patients, but only verbal consent was obtained. Due to the rarity of this condition and the inclusion of specific case details, the article content has been removed in order to avoid any potential privacy violations. The authors would like to apologize for their mistake as they believed that verbal consent was sufficient. 